\title{
El cuerpo como máquina: la aportación del dualismo cartesiano al desarrollo de la ciencia moderna
}

\author{
The body as a machine: cartesian dualism and its \\ contribution to modern science development
}

JOAN LLUÍS LLINÀS BEGON*

\begin{abstract}
Resumen: Este artículo defiende la importancia de la posición dualista de Descartes para el desarrollo de la ciencia moderna. Contrariamente al tópico que separa el Descartes científico del Descartes metafísico, y que considera éste como un rémora que impidió el correcto desarrollo de aquél, se sostiene la unidad y coherencia del pensamiento de Descartes, y el papel decisivo que tuvo, para constituir la ciencia moderna, la distinción cartesiana entre el alma y el cuerpo.

Palabras clave: Descartes, Dualismo cartesiano, Filosofía y ciencia del siglo XVII.
\end{abstract}

\begin{abstract}
This article defends the importance of Descartes dualist position for the development of modern science. Contrary to the approach that separates the Descartes as a scientist from the Descartes as a metaphysic, and that considers this later as an obstacle that prevented the proper development of Descartes as a scientist, in this paper I will argue for the unity and coherence of the Descartes' thought, and for its decisive role played for the contribution of modern science by his distinction between soul and body.
\end{abstract}

Keywords: Descartes, Cartesian Dualism, Philosophy and Science of XVII.

\section{Texto íntegro}

Este escrito parte del hecho indudable del influjo de Descartes en la cultura europea, y la percepción -por no decir también hecho- de la crisis del modelo teórico y práctico que surgió a partir del siglo XVII. Descartes fue un actor principal en la configuración de la llamada modernidad. Por eso mismo, la crisis de la misma parece afectar a quién fue uno de sus más decisivos impulsores. Parte también de la sensación que esa afectación mayormente ha supuesto una descalificación de la filosofía de Descartes, que se ha convertido en la diana

Fecha de recepción: 29/05/2016. Fecha de aceptación: 25/07/2016.

* Profesor Titular de Filosofía en la Universitat de les Illes Balears (UIB). Correo electrónico: jlluis.1linas@uib. es. Líneas de investigación: Filosofía Moderna, Montaigne, Descartes, Filosofía de la Educación. Publicaciones recientes: « La place de l'erreur dans la philosophie de Montaigne », Bulletin de la Société internationale des amis de Montaigne, $\mathrm{n}^{\circ}$ 63, 2016 - 1, p. 135-147 ; Montaigne, Ensayos sobre educación (editor), Madrid, Biblioteca nueva, 2015 ; «Educació, Filosofia i forma de vida. Les propostes de Montaigne i Descartes », Temps d'educació, $\mathrm{n}^{\circ}$ 46, 2014, p. 111-121. Este trabajo ha sido realizado en el marco del grupo de investigación sobre Pensamiento Científico y Filosófico Moderno y Contemporáneo (F\&C) de la Universitat de les Illes Balears (UIB). 
a la que hay que tirar antes de elaborar cualquier propuesta contemporánea, como aquello que hay descalificar, mostrando su falsedad, para poder construir algo nuevo. Obviamente, también se han dado movimientos para repensar a Descartes ${ }^{1}$. Sin embargo, en ese intento de "salvar" a Descartes se corre el riesgo de descuartizarlo para reivindicar sólo alguna pieza como válida. A uno de esos cortes me voy a referir ahora.

Como es bien sabido, entre los siglos XVI y XVII se produjo la llamada «primera revolución científica», en la que dos formas de entender el papel de la ciencia pugnaban por su hegemonía ${ }^{2}$. Por un lado el aristotelismo -y sus derivados escolásticos-, que presentaba una ciencia explicativa centrada en aspectos cualitativos. Por otro lado, la «ciencia moderna», que, tratando de superar explicaciones que apelaban lo cualitativo, postulaban la necesidad de considerar los aspectos cuantitativos, y centrada en la imagen de la máquina frente a la del organismo viviente. También es conocido que Descartes es considerado un agente principal en esta disputa y en la ulterior consolidación de la ciencia moderna. Ahora bien, si por una parte Descartes es visto como el fundamentador de un modelo -el mecanicismoque permitió el progreso de la ciencia moderna, por la otra se presenta su metafísica, y en concreto el planteamiento dualista del ser humano, como una rémora del pasado que debe -como de hecho se considera que ha sido- ser dejada atrás. Así, el Descartes moderno sería el científico, mientras que el Descartes metafísico sería el culpable del desarrollo de una modernidad condenada, en estos términos, al fracaso. Considero, ante esto, que un error de principio del planteamiento general acerca del papel de Descartes en el desarrollo de la nueva ciencia consiste precisamente en separar método, ciencia y metafísica en Descartes, como si fuesen compartimentos perfectamente aislables. Es decir, debemos evitar el anacronismo que supone afirmar que existen dos Descartes, uno "científico" y otro "metafísico"3. En el siglo XVII los que hoy denominamos "científicos" adoptaron compromisos teológicos o metafísicos que, sin embargo, no les impidieron hacer ciencia. En el caso de Descartes no debemos pasar por alto el carácter sistemático de su propuesta. La física cartesiana es metafísica en un sentido fuerte, pero no es solamente metafísica: los principios de la física (las leyes de la naturaleza), el marco teórico al cual debe ajustarse todo descubrimiento científico para Descartes, se derivan de la metafísica, pero las explicaciones científicas cartesianas son modelos mecánicos dirigidos a explicar fenómenos concretos.

1 En este sentido, es destacable la obra editada por Juan A. Nicolás y María José Frápolli, Evaluando la modernidad. El legado cartesiano en el pensamiento actual, Granada, Comares, 2001, un buen intento de repensar el cartesianismo, o también la edición a cargo de B. Bourgeois y J. Havet, L'esprit cartésien, Paris, Vrin, 2000, que recoge las ponencias de un congreso celebrado en París en 1996 en honor al cuarto centenario del nacimiento de Descartes.

2 Reducir la disputa a dos posiciones antagónicas es sin duda una simplificación. La ciencia moderna no aparece de golpe como una alternativa, sino que es fruto de un lento proceso, que empieza en el siglo XIV con unas investigaciones científicas teóricas que se van alejando del aristotelismo escolástico, y continua con posiciones como la magia y la filosofía natural, que en el siglo XVI se presentan como sendas alternativas a la ciencia escolástica. Para lo que nos interesa en este artículo, prescindiremos de este complejo proceso histórico y nos centraremos en la confrontación entre la "vieja" y la "nueva" ciencia, esto es, la confrontación entre la "vieja" y la "nueva" filosofía.

3 Anacronismo que no obstante está extendido entre algunos especialistas, como es el caso de Desmond Clarke, Descartes Philosophy of Science, Manchester, Manchester University Press, 1982. 
Una vez establecido esto, quiero centrarme en una cuestión: la modernidad del llamado dualismo cartesiano. Si asumimos que la física cartesiana es metafísica por sus principios (universales) pero es empírica por sus explicaciones (porque lo que interesa explicar son los fenómenos particulares, no todos los fenómenos posibles que podrían derivarse de dichos principios), entonces la pregunta es hasta qué punto la distinción establecida por Descartes entre alma y cuerpo es una rémora para el desarrollo de su física y, en concreto, de su fisiología mecanicista. Mi hipótesis al respecto es la siguiente: la teoría de la distinción establecida por Descartes fue, en el siglo XVII, una teoría consecuente con la nueva visión del mundo, y es moderna en dos sentidos:

1) Por una parte, la distinción permite que el cuerpo sea tratado separadamente del alma, y eliminar los entes superfluos de la Escolástica. El cuerpo puede ser explicado por sí mismo, sin necesidad de postular formas sustanciales o cualidades activas, y sin necesidad de recurrir al alma.

2) Por la otra, la consideración del hombre como un ser no exclusivamente mecánico permite que pueda elegir sus fines y llevarlos a cabo, con lo que la idea de progreso y de dominio de la naturaleza, propios de la modernidad, devienen posibles.

Centrándonos en el cuerpo vivo, en la actualidad se suele considerar que, en efecto, las posiciones dualistas que parten de Descartes fueron un obstáculo para el desarrollo de la ciencia, y en concreto para el estudio científico del ser humano. Contrariamente a esta posición, mantengo que las evidencias metafísicas (en concreto la distinción real) no sólo no fueron un obstáculo para el desarrollo de una fisiología mecanicista sino que supusieron un avance considerable con respecto al aristotelismo escolástico: el argumento de la distinción real nos muestra con certeza metafísica que los atributos del alma se restringen a las funciones del alma racional aristotélica y que los atributos del cuerpo vivo se amplían hasta abarcar las funciones del alma vegetativa y sensitiva aristotélica. Con esto se gana la posibilidad de explicar mecánicamente el funcionamiento del cuerpo vivo, abriendo el camino hacia lo que hoy llamaríamos una medicina científica. Así, en el Tratado del hombre, Descartes describe los hombres del nuevo mundo que explica. El inicio del Tratado es significativo:

Estos hombres estarán compuestos, como nosotros, de un Alma y un Cuerpo. Y hace falta que os describa, en primer lugar, el cuerpo aparte, y luego el alma también aparte; y en fin, que os muestre cómo estas dos Naturalezas deben estar juntas y unidas para componer hombres que se nos parezcan (AT XI, 119-120). ${ }^{4}$

4 «Ces hommes feront compofez, comme nous, d’vne Ame \& dVn Corps. Et il faut que ie vous décriue, premièrement, le corps à part, puis après, l'ame auffi à part ; \& enfin, que ie vous monftre comment ces deux Natures doiuent eftre iointes \& vnies, pour compofer des hommes qui nous reffemblent. ». Todas las referencias de la obra cartesiana se citan según la edición de referencia de Charles Adam y Paul Tannery (Oeuvres de Descartes, París, Léopold Cerf, 1897-1913; reimpresión París, Vrin, 1996). 
Tenemos, pues, tres realidades: el cuerpo, el alma, y la unión, y cada una debe ser tratada separadamente. En la misma línea, Descartes responde a Elisabeth de Bohemia, después que ésta le pregunte cómo algo inmaterial como el alma puede mover algo material como el cuerpo, lo siguiente:

Existen en nosotros ciertas nociones primitivas, que son como los originales en cuyo patrón nos basamos para formar todos nuestros conocimientos. Y esas nociones son muy pocas, ya que, tras las más generales, las del ser, el número, la duración, etc., que convienen para todo cuanto podemos concebir, sólo tenemos, en lo que más particularmente atañe al cuerpo, la noción de la extensión, de la que se derivan las de la forma y el movimiento. Y para el alma tenemos sólo la del pensamiento, que abarca las percepciones del entendimiento y las inclinaciones de la voluntad; y, por último, para el alma y el cuerpo juntos, sólo tenemos la de su unión, de la que depende la de la fuerza con que cuenta el alma para mover el cuerpo, y el cuerpo para influir en el alma, provocando en ella sensaciones y pasiones. ${ }^{5}$

Así pues, hay distinción, pero también unión alma-cuerpo. Y la noción de esta unión es tan primitiva como la del pensamiento y la de la extensión. Explicar cómo es posible esta aparente contradicción va más allá de la cuestión que nos ocupa aquí, y me limitaré a recordar que para Descartes no existe tal contradicción, aunque unión y distinción no puedan ser pensadas simultáneamente ${ }^{6}$. Esta dificultad de sostener simultáneamente la unión y la distinción fue uno de los principales problemas con los que se encontraron los continuadores del cartesianismo, y es una de las razones que condujo al rechazo más o menos generalizado del dualismo tal como Descartes lo formula. Posiciones más recientes consideran, desde una perspectiva histórica, que el dualismo cartesiano es una mera estrategia instrumentalista dentro de un juego retórico que tiene como finalidad última el progreso de la ciencia, en la línea de los estudios que recientemente han aparecido en torno a las relaciones de Descartes con la medicina ${ }^{7}$. En este sentido, la gran ventaja de la distinción consiste en no tener que apelar al alma para explicar el funcionamiento del cuerpo, como queda patente en el Tratado del hombre: El hombre máquina es un autómata capaz de formar representaciones cerebrales (en la glándula pineal) y de generar una respuesta adecuada a estas representaciones sin necesidad de que intervenga la mente. Para Descartes, la fundamentación de la

5 « Je considère qu'il y a en nous certaines notions primitives, qui sont comme des originaux, sur le patron desquels nous formons toutes nos autres connaissances. Et il n'y a que fort peu de telles notions; car, après les plus générales, de l'être, du nombre, de la durée, etc., qui conviennent à tout ce que nous pouvons concevoir, nous n'avons, pour le corps en particulier, que la notion de l'extension, de laquelle suivent celles de la figure et du mouvement; et pour l'âme seule, nous n'avons que celle de la pensé, en laquelle sont comprises les perceptions de l'entendement et les inclinations de la volonté; enfin, pour l'âme et le corps ensemble, nous n'avons que celle de leur union, de laquelle dépend celle de la forcé qu'a l'âme de mouvoir le corps, et le corps d'agir sur l'âme, en causant les sentiments et les passions » (AT, III, 665; trad. esp. María Teresa Gallego Urrutia, Descartes, Madrid, Gredos, 2011, pp. 553-554).

6 Para un desarrollo de esta cuestión, véase J.L. Llinàs, "La integración de la experiencia de la unión en la filosofía", en De la Metafísica a la Antropología. Reinterpretando el dualismo de Descartes. Ed. François Jaran, València, Pre-textos, pp. 39-52, 2014.

7 Véase por ejemplo Gideon Manning, "Descartes and the Bologna Affair", The British Journal for the History of Science 47, pp. 1-13, 2014. 
nueva ciencia no puede pasar por considerar al ser humano como una unidad substancial. Descartes quiere evitar una fisiología que considere atributos corporales y mentales, pues esta es precisamente una fisiología de base aristotélica, una fisiología que explica determinadas funciones apelando a causas anímicas. Si no hay lugar para cualidades anímicas en fisiología, y las cualidades de los cuerpos pueden explicarse exclusivamente en términos de materia y movimiento, entonces distinguir y separar alma y cuerpo desde la metafísica se revela como una estrategia adecuada para fundamentar dicha fisiología.

Ahora bien, Descartes podía haber adoptado posiciones diversas y alternativas a la aristotélica escolástica. Por una parte, podría haber adoptado una posición similar a la de Montaigne, que vindica el cuerpo en un esquema de unión alma-cuerpo. Sin embargo, Montaigne no intenta fundamentar la ciencia, y su indudable modernidad en la defensa del ser humano como un ser corporal queda matizada al mantener una posición escéptica en relación a la posibilidad de obtener verdades sobre el mundo que hagan posible el progreso científico. Por la otra, podría haber adoptado una posición materialista. Al respecto, cabe recordar que el dualismo cartesiano no fue una posición globalmente aceptada en el siglo XVII. Más allá de las propuestas correctivas hechas desde corrientes racionalistas (ocasionalismo, armonía preestablecida), el materialismo se presentaba como una teoría alternativa, conforme a la nueva ciencia, bastante extendida. Esto es así incluso en el campo del llamado cartesianismo. Un ejemplo de ello es Regius, que se consideraba seguidor de Descartes, aunque su interpretación de la filosofía cartesiana condujo a un enfrentamiento entre ambos. Para lo que nos ocupa, hay que destacar que la posición de Regius conduce a una consideración material del alma, en la medida que el alma es triple, incluyendo la fuerza vegetativa y la motriz, a lo que se opone Descartes, que considera que estas fuerzas se reducen, en el hombre, a la disposición que tienen las partes del cuerpo de mover y ser movidas ${ }^{8}$.

Más sólidas son las objeciones de Hobbes a la doctrina de la distinción real entre mente y cuerpo, pues muestran que es posible mantener una posición estrictamente materialista dentro de la nueva filosofía sin sostener una posición dualista9 ${ }^{9}$. Hobbes sostiene que el cogito no prueba la existencia de un alma inmaterial, sino al contrario. Esto es así porque distinguimos entre el sujeto y las facultades o accidentes que residen en ese sujeto, lo que impide que llevemos a cabo la inferencia "Yo estoy pensando, entonces yo soy pensamiento", inferencia tan absurda como "Yo estoy paseando, entonces soy un paseo"10. Tenemos que atribuir, pues, un sujeto de los accidentes y facultades relativos al pensamiento, y es perfectamente razonable considerar que ese sujeto es el cuerpo. No voy a entrar en esa discusión, que en último término remite a la existencia o no de ideas innatas, sino que, independientemente de a quién demos la razón, la posición de Hobbes es lo suficientemente consistente como para mantener una postura estrictamente materialista sobre el mundo. Y, si pensamos el sujeto como materia, la ventaja 1) del dualismo a la que antes aludíamos está solucionada. Si todo es materia, entonces podemos llevar a cabo la investigación del mundo natural sin los inconvenientes del animismo.

8 Véase Laura Benítez, "La vía de reflexión epistemológica y el dualismo cartesiano", en: La modernidad cartesiana, Santiago de Cali, Universidad del Valle, 2013, pp. 17-27.

9 Véanse las Terceras Objeciones a las Meditaciones Metafísicas, AT VII, 171-196; AT IX, 133-152.

10 AT VII, 172-173. 
Entonces, o bien renunciamos a plantear la cuestión en términos de utilidad y sostenemos que la distinción cartesiana es una consecuencia del despliegue de su metafísica, que es independiente del desarrollo de la ciencia, o bien, si queremos sostener que, más allá de la convicción cartesiana respecto a la distinción, ésta no va "contra los tiempos", debemos reformular la pregunta en los siguientes términos: ¿Qué ventaja adicional presenta el dualismo respecto del materialismo en relación a la constitución de la modernidad? No me refiero, pues, a por qué Descartes adoptó una posición dualista, sino a la ventaja que supone su adopción en un contexto en la que se configura una nueva visión del mundo. Aparentemente es un inconveniente, pues parece suponer la escisión del hombre en dos. Pero este inconveniente queda matizado, como ya he señalado, por la defensa de la unión cuerpo-alma. El verdadero hombre es el resultado de esa unión, por lo que, aunque alma y cuerpo sean separables, en el ser humano el alma no funciona separada de un cuerpo, sino que está unida a él de una manera, nos dice Descartes, más fuerte que la que une al piloto con su navío.

En este sentido, hay que recordar que el árbol de la filosofía, tal como es explicado en la carta-prefacio a la edición francesa de Los principios de la filosofía, se dirige a que el hombre recoja los frutos del árbol ${ }^{11}$. El saber es único, todo está conectado, pero ni la metafísica (las raíces) ni la física (el tronco), adquieren su sentido sin unas ramas que den frutos. Y esos frutos los recoge el verdadero hombre, ese hombre unión de dos sustancias. La filosofía ya no es mera contemplación, ya no se trata del saber por el saber, sino de un "saber para". Pero lo característico del proyecto moderno es que este "saber para" viene definido por el propio ser humano ${ }^{12}$. Las ramas del árbol de la filosofía dejan patentes las finalidades que se impone el ser humano: la conservación de la vida (medicina), la disminución del trabajo humano (mecánica), y la satisfacción (moral). Estas finalidades dan sentido al desarrollo técnico, que en su despliegue no debe olvidar porqué se desarrolla. Para lo que nos ocupa, quiero resaltar que, en la filosofía cartesiana, si el ser humano se marca sus propios fines, y si puede, en cierto modo, ocupar el lugar de Dios o de la naturaleza, es porque posee un alma inmaterial y, por tanto, no sometida al mecanicismo de la res extensa. Una mente con voluntad libre, que puede determinar sus propios fines y actuar en consecuencia. Se trata de la ventaja 2) a la que aludíamos al principio, ventaja que un modelo materialista no proporciona tan fácilmente, pues todo parece reducirse a relaciones causa-efecto. La cuestión última que está en juego, por tanto, es el de la libertad. Un modelo estrictamente mecanicista conduce a redefinir el concepto de libertad humana, que, en última instancia, no puede oponerse o desviarse de la necesidad que impone la cadena causal. El planteamiento cartesiano permite preservar la libertad humana en la medida que el ser humano no está sometido a ninguna fuerza externa que le conduzca a una opción determinada, sino que puede elegir la opción sin condicionamiento externo. En este sentido, el hombre se asemeja a Dios y le sustituye, ya que en el nuevo modelo de ciencia desconocemos los fines últimos de la naturaleza, fines que quedan fuera de nuestro alcance, por lo que, para dar sentido a la realidad, no queda más remedio que el hombre genere sus propios fines. Esto no significa que el hombre sea absolutamente libre

11 AT IX, 14.

12 La sexta parte del Discurso del método (AT VI, 60ss) deja patente la idea de progreso ligada a la empresa colectiva de desarrollo de la nueva ciencia. 
como lo sería Dios, pues el alma está unida a un cuerpo e interacciona con él. Es decir, en tanto que el alma se distingue del cuerpo, el hombre es libre; pero en tanto que está unida al cuerpo, está condicionada por éste. En tanto que el alma se distingue del cuerpo, puede elaborar sus propios fines; en tanto que está unida al cuerpo, puede llevar a cabo este diseño.

En consecuencia, la distinción cartesiana entre alma y cuerpo, que va acompañada de la defensa de la unión cuerpo-alma, aparece como la teoría más eficaz para consolidar dos de los principales aspectos que configuran la naciente modernidad del siglo XVII: el desarrollo de la nueva ciencia (frente a la escolástica), y el establecimiento de los propios fines (frente al materialismo). La confluencia de estos dos aspectos revela que la nueva ciencia no supone únicamente una visión del mundo verdadera frente a la teoría falsa anterior, sino que abre la puerta a considerar la ciencia como un buen instrumento para mejorar la vida del ser humano. Esto no significa que ese fuese el "auténtico" objetivo de Descartes, ni que la teoría de la distinción sea una mera teoría instrumentalista, una retórica eficaz para los fines propuestos. La teoría pretende reflejar un estado de cosas: la metafísica es una parte del sistema, son las raíces del árbol, y la distinción se presenta como necesaria para la demostración de la existencia del mundo externo y, paradójicamente, de la consideración del hombre como uno. Pero su pretensión de verdad es un asunto diferente de los efectos que produce sobre la constitución del pensamiento moderno. En este sentido, la posición de Descartes sobre la mente y el cuerpo supone una contribución valiosa para consolidar la modernidad en dos de sus aspectos fundamentales: la nueva ciencia y la consideración del hombre como generador de fines que den sentido a ese desarrollo.

\section{Referencias bibliográficas}

Benítez, L. (2013): "La vía de reflexión epistemológica y el dualismo cartesiano", en: $L a$ modernidad cartesiana, Santiago de Cali, Universidad del Valle, pp. 17-27.

Bourgeois, B. \& Havet, J. (2000) : L'esprit cartésien, Paris, Vrin.

Clarke, D. (1982): Descartes Philosophy of Science, Manchester, Manchester University Press.

Descartes, R. (1996): Oeuvres, Ed. Adam, C. \& Tannery, P., reimpresión París, Vrin (1ª edición París, Léopold Cerf, 1897-1913).

Descartes, R. (2011): Reglas para la dirección del espíritu [y otras obras], estudio introductorio de Cirilo Florez, Madrid, Gredos.

Llinàs, J.L. (2014): "La integración de la experiencia de la unión en la filosofía", en De la Metafísica a la Antropología. Reinterpretando el dualismo de Descartes. Ed. François Jaran, València, Pre-textos, pp. 39-52.

Manning, G. (2014): "Descartes and the Bologna Affair", The British Journal for the History of Science 47, pp. 1-13.

Nicolás, J.A. \& Frápolli, M.J. (2001): Evaluando la modernidad. El legado cartesiano en el pensamiento actual, Granada, Comares. 
